\title{
ULTRA-FAST SPIN DYNAMICS IN DILUTED MAGNETIC SEMICONDUCTORS*
}

\author{
Z. Wilamowski, R. Buczko, G. KarcZewsiki \\ Institute of Physics, Polish Academy of Sciences \\ Al. Lotników 32/46, 02-668 Warszawa, Poland \\ AND W. JANTSCH \\ Johannes Kepler Universität, 4040-Linz, Austria
}

In nano-size antiferromagnetic systems a spatially inhomogeneous field leads to the formation of a staggered magnetization. Thereby the total magnetic moment does not change but the formation of a net magnetic moment at the border of the cluster leads to an energy gain. This type of magnetism is characterised by an ultra-fast dynamics. We suggest it is also responsible for the formation of the exciton magnetic polaron.

PACS numbers: $75.10 . \mathrm{Jm}, 75.50 .-\mathrm{y}$

A few recently discovered effects in diluted magnetic semiconductors are still not fully understood as yet. In particular, the characteristic time for the creation of an exciton magnetic polaron (EMP) is extremely short. It is much faster than any longitudinal relaxation time [1-3]. In addition, there is a "mysterious" coupling between antiferromagnetic (AF) layers in antiferromagnet-semiconductor superlattices [4] and also between antiferromagnetic micro-grains which are characterised by a new type of magnetic resonance [5]. In this paper we suggest that for the explanation of this type of phenomena one has to consider the response of the magnetic system on a local, spatially inhomogeneous perturbation. The discussed effect has a pure quantum character therefore we analyse a rigorous solution of the Hamiltonian

$$
\mathcal{H}=\sum_{i, j} J_{i, j} S_{i} S_{j}+\sum_{i, j} D_{i, j}\left(3 S_{i, z} S_{j, z}-S_{i} S_{j}\right)
$$

The first term in Eq. (1) describes the exchange energy, the second some magnetic anisotropy. We examine the solutions for linear chains rigorously. This is the simplest possible case where the above-mentioned phenomena can be demonstrated. Moreover, we limit our discussion to chains with an even spin number, $N$. For odd spin number the ground state is characterised by a $2 S+1$ degeneracy, i.e., by a nonvanishing magnetic moment. In that case, the discussion of the magnetic properties is more complicated since the Curie magnetism caused by the magnetic

*This work was supported by the Committee for Scientific Research (Poland) grant 2 P03B 19108 and by Fonds zur Förderung der Wissenschaftlichen Forschung (Austria). 
moment of the ground state interferes with the staggered magnetism caused by inhomogeneous magnetic fields.

The quantum mechanical calculation of finite AF clusters [6] shows that for an even number of spins, $N$, for axial symmetry and positive anisotropy parameter $D>0$, a non-magnetic singlet $(M=0)$ is a ground state of the cluster. The first excited state, separated by an energetic distance, $\Delta$, is another non-magnetic singlet. These states determine the magnetic properties of a finite size AF. A crucial point is that the mean values of all possible spin components vanish for these states, i.e., no external field can lead, within the first order perturbation, to the formation of any local magnetic moments. They can appear, however, when a second-order perturbation is considered.

The dominant second-order effect is caused by a mixing of two singlets. But since they are of different symmetry (due to parity symmetry for exchange of equivalent sites) only an antisymmetric perturbation by external fields

$$
\mathcal{H}_{\mathrm{p}}=g \mu_{\mathrm{B}} \sum_{i} \boldsymbol{H}_{i} \boldsymbol{S}_{i}
$$

leads to nonvanishing matrix elements

$$
p=\left\langle\psi_{a}\left|H_{\mathrm{p}}\right| \psi_{s}\right\rangle=g \mu_{\mathrm{B}} \sum_{i} \boldsymbol{H}_{i}\left\langle\psi_{a}\left|S_{i}\right| \psi_{s}\right\rangle \equiv g \mu_{\mathrm{B}} S_{\mathrm{ch}}(\boldsymbol{H} \cdot \boldsymbol{\sigma}) .
$$

The matrix elements vanish for spatially homogeneous perturbations (constant $H_{i}$ ), therefore they lead neither to the formation of local moments, when a static perturbation is considered, nor to any resonant transition, when time dependent fields are applied.

The magnetic properties of finite AFs are determined by their response to spatially inhomogeneous effective fields. Such perturbations mix two singlets which lead to the formation of a staggered magnetization. The total magnetization remains zero but finite static local moments are created. These are antiparallel in the two AF sublattices. At the ground state the spatial distribution of magnetic moments is determined by the matrix element of the spin operator (Eq. (2)) between states of unperturbed AFs, and thus it does not depend on the character nor the amplitude of perturbation. It is described by a normalised vector $\sigma_{\mathrm{ch}}\left(\sum_{i} \sigma_{i}^{2}=1\right)$, which is defined in Eq. (3) and it characterises the only possible way to form a staggered magnetization. For a Heisenberg chain $(D=0)$ the amplitudes, $\sigma_{i}$, are not equal among each other, but they are antiparallel. With increasing axial symmetry $(D>0)$ the amplitudes of the local magnetization become equal and for Ising anisotropy $(D=J)$, the individual spin magnetizations become of a regular staggered shape

$$
S_{\mathrm{ch}} \sigma_{i}=\frac{(-1)^{i} S}{\sqrt{N}}
$$

The change of the ground state energy decreases with increasing perturbation

$$
\Delta E_{0} \approx-\frac{2 p^{2}}{\Delta}=-\frac{2\left(g \mu_{\mathrm{B}} S_{\mathrm{ch}}\right)^{2}}{\Delta}(\boldsymbol{\sigma} \cdot \boldsymbol{H})^{2} \equiv-\frac{1}{2} \chi_{\mathrm{g}} H_{\mathrm{p}}^{2} \equiv \frac{1}{2} \boldsymbol{\mu} \cdot \boldsymbol{H}
$$

where $H_{\mathrm{p}}=\boldsymbol{\sigma} \cdot \boldsymbol{H}$ is a projection of the distribution of magnetic fields, on the characteristic vector. The change of energy can be interpreted as the gain caused by an induced characteristic magnetization 


$$
\boldsymbol{\mu}=-g \mu_{\mathrm{B}}\left\langle\boldsymbol{S}_{i}\right\rangle=\chi_{\mathrm{g}}(\boldsymbol{\sigma} \cdot \boldsymbol{H}) \boldsymbol{\sigma}=\chi_{\mathrm{g}} H_{\mathrm{p}} \boldsymbol{\sigma} .
$$

The effective mean value of $\left\langle S_{i}\right\rangle$ is half of that calculated as a quantum mechanical mean value of the operators $S_{i}$ at the ground state. This difference is caused by the fact that half of the gain of the Zeeman energy is compensated by the "staggered energy" - the reduction of the exchange energy which is needed to create the characteristic magnetization. Since the energy splitting is of the order of $\Delta=\alpha J / N$ (where $\alpha \approx 10$ depending on $S$ and the cluster type) the generalised susceptibility of the whole cluster is described by

$$
\chi_{\mathrm{g}}=\frac{4\left(g \mu_{\mathrm{B}} S_{\mathrm{ch}}\right)^{2}}{\Delta} \approx\left[\frac{\left(g \mu_{\mathrm{B}} S_{\mathrm{ch}}\right)^{2}}{3 J}\right] \frac{12}{\alpha} N
$$

The expression in the square brackets is equal to the Curie-Weiss susceptibility of a single spin at zero temperature and $\alpha \approx 12$. Therefore, in order to compare the energy gain caused by the induction of staggered (characteristic) susceptibility with the gain caused by the classical Curie magnetism, one has to analyse the ratio, $r$, of the square of the projection $H_{\mathrm{p}}$ and the mean value of square of local fields. For the purpose of this analysis we assume that the characteristic vector is described by Eq. (4). For a simplest case, when the effective field acts on the first (surface) spin only, the contribution to the energy gain due to the Curie-Weiss magnetization of the first spin is equal to the gain caused by the induction of the staggered magnetization in the whole AF cluster, $r=1$. For semiconductor/AF superlattices the barrier is built from an insulating, large gap AF. Thus the contributions to the $s p-d$ coupling between free carrier in semiconductor well with spins localised in AF barrier caused by the two types of magnetism are comparable. When the penetration of an exciton in the AF barrier is deeper, the effect of the staggered magnetization becomes smaller and vanishes when the exciton Bohr radius in the barrier becomes longer than a characteristic size: the thickness of the AF layer or the size of clusters in diluted AF.

Another extreme case occurs if the effective field has a staggered character. The hyperfine field which originates from nuclear spins, polarised by a static AF magnetization has this type of spatial distribution. The staggered susceptibility of the $\mathrm{AF}$ cluster increases linearly with the size of cluster, $N$. In that case, $r=1$ and the contribution to the energy gain caused by the "staggered magnetism" is equal to that caused by the Curie magnetism.

Generally, the appearance of the staggered magnetization does not change the total magnetic moment of the AF cluster but it leads to the formation of net magnetic moments at the cluster surface, i.e., to the creation of a quadrupole magnetic moment which leads to a gain in the coupling energy to the inhomogeneous field. The energy gain caused by this mechanism is, usually, smaller as compared to the energy gain caused by the Curie-Weiss magnetization. It becomes of similar magnitude for very small $\mathrm{AF}$ where at least one dimension is reduced to a few inter-atomic distances, like in nano-AF grains [3], or AF/semiconductor superlattices [2,4] or diluted magnetic semiconductors [4] which can be treated as a set of random mesoscopic AF clusters.

The basic difference between the two types of magnetism is the very different range of characteristic times. In DMS, at liquid helium temperature, longitudinal 
relaxation times related to the spin flips are spread from the range of milliseconds down to, for very diluted impurities, $10^{-7} \mathrm{~s}$ for dense DMS [1]. At lower temperatures they become longer and diverge at zero temperature. In contrast, the formation of the staggered magnetization does not need any quantum transition (spin flips). In that case the perturbation "immediately" modifies the quantum state, and thus the dynamics of the response of AF cluster is limited by the characteristic frequency for oscillations of the staggered magnetization. This frequency is determined by the energy distance $\Delta=\alpha J / N$. For $\alpha=10, J / k_{\mathrm{B}}=10 \mathrm{~K}$, the characteristic frequency is expected to be $\omega=10^{13} \mathrm{~s}^{-1} / N$. The resonance observed by Awschalom [5] in small 3D AF grains directly corresponds to this characteristic frequency. For $N \approx 10^{3}-10^{4}$, the resonance is observed at $\omega \approx 10^{9}-10^{7} \mathrm{~s}^{-1}$. This type of magnetism is much faster, also for mesoscopic size of the AF cluster. Moreover, the characteristic frequency is not temperature dependent. Thus it determines the fast dynamics at low temperature.

The binding energy of the static, bound magnetic polarons is predominantly caused by a classical magnetization of localised spins. But Curie-Weiss magnetism cannot be responsible for the binding of EMP where the exciton lifetime is in the ps range. In that case the dominant contribution to the energy seems to be caused by the formation of the staggered magnetization. In this case the total magnetic moment of clusters does not change. The only effect is the formation of a local magnetization in the cluster volume, where the perturbation field is biggest, and a "diffusion" of antiparallel magnetization to regions of lower fields. Thus, it is clear, without any detailed analysis, that for a random set of AF clusters a considerable effect of the binding of EMP occurs when the characteristic length of the clusters is larger than the exciton Bohr radius. Such behaviour is well confirmed by the experimental data [2,3] - a non-zero binding of EMP appears for alloy compositions $x>0.1$, i.e., for alloys where the mean size of the AF cluster becomes comparable to the Bohr radius. For smaller composition no EMP effect is observed; clusters are smaller than the exciton size.

The coupling between antiferromagnetic layers via an intercalated semi-insulating diamagnetic layer is too weak to be responsible for the static correlation of spins among the AF layers. But this type of coupling is strong enough to provide a dynamical correlation among oscillating resonators. Thermal neutrons are so fast that they monitor only the momentary spin correlations.

\section{References}

[1] T. Dietl, P. Peyla, W. Grieshaber, Y. Merle d'Aubigné, Phys. Rev. Lett. 74, 474 (1995).

[2] D.R. Yakovlev, Acta Phys. Pol. A 90, 703 (1996).

[3] G. Mackh, W. Ossau, D.R. Yakovlev, A. Waag, G. Landwehr, R. Hellmann, E. Göbel, Phys. Rev. B 49, 10248 (1994).

[4] T. Giebultowicz, V. Nunez, G. Springholz, G. Bauer, J. Chen, M.S. Dresselhaus, J.K. Furdyna, J. Magn. Magn. Mater. 140-144, 635 (1995).

[5] D.D. Awshalom, J.F. Smyth, G. Grinstein, D.P. DiVincanzo, D. Loss, Phys. Rev. Lett. 68, 3092 (1992).

[6] R. Buczko, Z. Wilamowski, W. Jantsch, Acta Phys. Pol. A 90, 747 (1996). 